\title{
Hypovitaminosis D in Child Population
}

\author{
M. Popovicova (Maria Popovicova)1, M. Belovicova (Maria Belovicova)1, 2, 3, \\ T. Hudakova (Tatiana Hudakova) ${ }^{1}$
}

${ }^{1}$ St. ElizabethUniversity of Health and Social Sciences, Bratislava, SK.

${ }^{2}$ Internal Clinic for Liver Disease Diagnosis and Treatment, Remediums.r.o.,

Bardejov, SK.

${ }^{3}$ Slovak Society of Practical Obesitology (SSPO), Bardejov, SK.

\section{E-mail address:}

majapopovicova7361@gmail.com

\section{Reprint address:}

Maria Popovicova

Pupavova 4

Michalovce

07101

Slovakia

Source: Clinical Social Work and Health Intervention

Pages: $40-45$

Volume: 12

Issue: 3

\section{Reviewers:}

Michael Costello

University of Scranton School of Education, USA

Gabriela Lezcano

University of California, San Francisco, USA

\section{Keywords:}

Vitamin D. Hypovitaminosis D. Prevention, Pediatricpatient. Endocrinology.

\section{Publisher:}

International Society of Applied Preventive Medicine i-gap

CSWHI 2021; 12(3): 40 - 45; DOI: 10.22359/cswhi_12_3_07 CC Clinical Social Work and Health Intervention

\section{Abstract:}

Introduction: Hypovitaminosis D is a global problem in both, children and adults. At low vitamin D levels bone deformities, growth retardation; as well as increased susceptibility to infectious and autoimmune diseases have been observed in children. Due to its multiple systemic effect, the importance of vitamin $\mathrm{D}$ is irreplaceable in development of a child's organism.

The sample and aim of the research: We approached parents of 181 children who visited endocrinological and osteological outpatient clinics in Eastern Slovakia. The aim of the research was to determine the status of hypovitaminosis D in pediatric patients. We investigated the relationship between the lifestyle of a child and the occurrence of hypovitaminosis D. At the same time, we mapped parents' knowledge regarding preven- 
tion of hypovitaminosis $\mathrm{D}$ development being conducted at pediatric outpatient clinics.

Methods: To verify the hypotheses, we used inductive statistical tools - the independent T-test and Chi - square test. Based on the calculated value and the significance level of 0.05 , we made decisions about the significance of the differences.

Results: By deeper analysis we found that hypovitaminosis D is more common in children with endocrine disorders. The relationship between the incidence of hypovitaminosis D and metabolic disease was not statistically significant. Parental knowledge of appropriate ways of hypovitaminosis D prevention is related to the occurrence of the disease. No significant relationship between the child's lifestyle and the occurrence of hypovitaminosis $\mathrm{D}$ has been confirmed.

Conclusion: The occurrence of vitamin D deficiency has an adverse effect on human health. It is important to pay particular attention to this issue at the primary prevention level. We also consider it important for the parent to lead the child to an active lifestyle and to responsibility for their own health.

\section{Introduction}

\section{„A house not seen by the sun is seen by the doctor."}

This well-known folk proverb, historically proven and concise, conceals a great wisdom. As early as 1822 , Dr. Sniadecki noticed for the first time the relationship between the effects of sunlight and the occurrence of rickets. He pointed out the benefits of sunbathing and the need for vitamin D (1). However, nowadays experts' opinion on this topic is divided. On the one hand there are the beneficial effects of sunlight on the synthesis of vitamin D in the fight against the vitamin $\mathrm{D}$ deficiency pandemic, and on the other hand, the negative effect of excessive sunlight and the need for protection using products with high sun protection factor are pointed out. Since long ago, the effects of Vitamin D apply to the skeletal muscle system. This view is refuted by numerous studies which, in addition to the main role of vitamin D - the regulation of calcium and phosphorus metabolism - confirm its other effects on the body. These include the beneficial effects of vitamin D on the immune system; on the incidence of autoimmune diseases; on insulin secretion; on the effect on the incidence of obesity and vascular disease.

Due to its multiple systemic effect, the importance of vitamin D is irreplaceable in devel- opment of child's organism. At a low vitamin D level, bone deformities, growth retardation as well as increased susceptibility to infectious and autoimmune diseases have been observed in children.

By adapting to a modern way of life, when one spends a significant part of the day indoors and leads not very active lifestyle, globally, we have come to the pandemic of vitamin D deficiency over the past 20 years, both in the adult population and in children, which is confirmed by numerous international studies. 1As reported by Furkova (2010), up to $77.7 \%$ of Slovak children have vitamin D deficiency. A sensitive period for the development of a low level of vitamin $\mathrm{D}$ due to a period of rapid and intensive growth is the neonatal period, infancy and puberty. Currently, children over 1 year of age and adolescents are the most sensitive population for the occurrence of low vitamin D levels (2).

\section{Hypovitaminosis D}

The influence of modern times, which brought about a change of lifestyle and ways of eating has left its signature in form of an epidemic and even a pandemic of low vitamin D levels in adults and in children in both, developed and developing countries. It has been estimated that about one billion people worldwide have hypovitaminosis D. A Boston study conducted at the end of winter shows the occurrence of low 
vitamin $\mathrm{D}$ concentrations in two thirds of the healthy young population (3). As Furková stated in her study, hypovitaminosis D was found in up to $77.7 \%$ of the Slovak pediatric population. Rickets has also started to reoccur (4). Due to the increased requirements of the body during the period of growth and development, children present a sensitive group at risk of developing hypovitaminosis D. Prevention of vitamin D deficiency seems to be important not only in infants, but especially in older children.

Table 1: Criteria for the evaluation of serum vitamin D concentrations (2).

\begin{tabular}{|c|c|c|c|}
\hline \multirow[t]{3}{*}{$\begin{array}{l}\text { Vitamin D } \\
\text { status }\end{array}$} & \multicolumn{3}{|c|}{$\begin{array}{l}\text { Serum calcidiol } \\
\text { concentration }\end{array}$} \\
\hline & \multicolumn{2}{|c|}{$\begin{array}{l}\text { According } \\
\text { to Bordelon }\end{array}$} & \multirow{2}{*}{\begin{tabular}{|c}
$\begin{array}{c}\text { According } \\
\text { to IOM }\end{array}$ \\
$\mathrm{ng} / \mathrm{ml}$ \\
\end{tabular}} \\
\hline & $\mathrm{ng} / \mathrm{ml}$ & $\mathrm{nmol} / \mathrm{l}$ & \\
\hline $\begin{array}{l}\text { Severe } \\
\text { deficiency }\end{array}$ & $\leq 10$ & $<25$ & $\leq 10$ \\
\hline Deficiency & $<30(10-20)$ & $<75$ & $\leq 20$ \\
\hline Insufficiency & $21-30$ & $50-75$ & $21-29$ \\
\hline Optimal values & $\geq 43$ & $\geq 75(100)$ & $\geq 40$ \\
\hline $\begin{array}{l}\text { Toxic } \\
\text { concentrations }\end{array}$ & $\geq 150(100)$ & $\geq 340$ & \\
\hline
\end{tabular}

A reduction in serum calcidiol concentrations below $30 \mathrm{ng} / \mathrm{ml}(75 \mathrm{nmol} / \mathrm{l})$ is therefore assessed as hypovitaminosis D - vitamin D deficiency in general (5).

\section{Aim of the research}

The main aim of the research was to point to an increased incidence of hypovitaminosis D in the pediatric population. We examined the relationship between the lifestyle of the child and the occurrence of hypovitaminosis D. We mapped parents' knowledge regarding prevention of hypovitaminosis $\mathrm{D}$ development that is being conducted at pediatric outpatient clinics. We focused on finding associations between the incidence of hypovitaminosis $\mathrm{D}$ in pediatric patients with endocrine and metabolic diseases. We also wanted to determine the incidence of hypovitaminosis D in connection with BMI index values.

\section{Research sample and methodology}

Our research was performed on a sample of patients from two specialized outpatient clinics a pediatric endocrinology outpatient clinic and an osteological outpatient clinic. The data on vitamin D levels and information about decreased bone density measurements were obtained from a secondary source from the parents of children. The primary data are the measured values given in the patient's medical records. The survey included 181 respondents. In 56 children of the respondents the vitamin D value was normal; 63 respondents reported insufficiency; 62 respondents reported hypovitaminosis D. The hypotheses were verified by inductive statistics - statistical tests - Chi - square test and unpaired $\mathrm{t}$ - test. We used a significance level of 0.05 .

\section{Results}

We assumed that there was a significant relationship between the parent's knowledge regarding the prevention of hypovitaminosis D and its occurrence. Between information on the hypovitaminosis D prevention and decreased levels of vitamin D in the child's blood, a statistically significant difference was found which means that the awareness of the child's parents regarding hypovitaminosis $\mathrm{D}$ prevention is associated with the occurrence of the disease. The validity of the hypothesis that there is a significant relationship between the lifestyle of the child and the occurrence of hypovitaminosis D was verified by several sub-hypotheses. Exercise improves overall blood flow in the body, function of the respiratory system, cardiovascular system, vascular system of lower limbs, and last but not least, of the nervous system (6). No statistically significant relationship between regular sporting / physical activity and the presence of hypovitaminosis D was found. No statistically significant difference between children with a dairy rich diet and children with a low dairy intake and the presence of hypovitaminosis D was confirmed. However, between the amount of time spent in fresh air and daylight and the presence of hypovitaminosis D a statistically significant relationship was confirmed. We also tested the relationship between the child's gender variable and the incidence of hypovitaminosis $\mathrm{D}$. The calculated value is less than the significance level of 0.05 . This means that there is a significant relationship between the 
occurrence of hypovitaminosis D and the gender of the child. The incidence of hypovitaminosis D is more common in girls than in boys. The relationship of hypovitaminosis D in relation to the occurrence of endocrine disorders is not statistically significant. In terms of endocrine disorders, we observed the presence of any one or more of the diseases: primary hyperparathyroidism, thyroiditis or diabetes mellitus. The occurrence of metabolic disorders was also significantly represented in respondents without the presence of hypovitaminosis D. Under metabolic disorders, we observed the presence of any one or more of the diseases: obesity, elevated cholesterol levels or chronic inflammatory bowel disease. We assumed that the incidence of hypovitaminosis D was associated with higher BMI levels. This relationship was confirmed, so the incidence of hypovitaminosis D is related to higher BMI values. In addition, we tested the relationship between hypovitaminosis $\mathrm{D}$ and measured decreased bone density in children. We confirmed the relationship between the occurrence of decreased bone density and hypovitaminosis D.

\section{Discussion}

Recently, the topic of vitamin D has been frequently mentioned due to the high prevalence of its deficiency in the population. Decreased levels of vitamin D are a problem in children in developed and developing countries. Vitamin D is a trigger and regulator of hormonal processes essential for life. There are scientific publications pointing at a significant relationship between the occurrence of certain diseases and lowered vitamin D levels.

181 respondents from 2 specialized outpatient clinics - a pediatric endocrinology and an osteological outpatient clinic - were included in the research. The main aim of our research was to detect the incidence of hypovitaminosis $\mathrm{D}$ in pediatric patients. 62 respondents reported hypovitaminosis D; 63 respondents reported insufficiency. A statistically significant relationship was found between the amount of time spent in the fresh air and daylight and the presence of hypovitaminosis D. We also found a link between parental awareness of the prevention of hypovitaminosis D development and the incidence of the disease in children. BMI values are associated with hypovitaminosis D. We also tested the relationship be- tween hypovitaminosis $\mathrm{D}$ and the detected decreased bone density in children. This assumption has been confirmed.

According to the results of a Chinese study, half of the Chinese healthy population had low levels of vitamin D (25-50 nmol / 1). The most represented category was the group of females of age $18-39$ years $(66.3 \%$ vs. males $45.3 \%$, p $<0.01)$. Low levels were associated with higher concentrations of parathyroid hormone, phosphates and lower calcium levels. A cross-section of several recent European studies has indicated that vitamin D supplementation in infants during the first year of life may prevent the development of type 1 diabetes. A dose of $50 \mu \mathrm{g} /$ day was associated with a reduced risk of diabetes in Finland, but the efficacy of lower doses has not been studied. The recommended dietary intake of vitamin D for children in the United States is $5 \mu \mathrm{g}$ / day and the upper level is $25 \mu \mathrm{g} /$ day. There is no evidence that intake between 5 and $25 \mu \mathrm{g} /$ day would reduce the incidence of diabetes, but it seems beneficial to ensure that infants reach at least a lower vitamin D intake per day. Experimental studies have shown that vitamin D supplementation is associated with a decrease in fasting blood glucose as well as with an improvement in insulin sensitivity in patients with pre-diabetes.

Hypovitaminosis D has been associated with the development of obesity glucose intolerance; progressive development of metabolic syndrome. In deficiency of vitamin $\mathrm{D}$ the apoptosis of $\beta$ cellsin islets of Langerhans has beenproved. Vitamin D deficiency inhibits insulin secretion (2). In 489 people, Kayaniyl (2011) found that higher concentrations of vitamin D, independent of other factors, improved pancreatic beta cell function and the glycemic curve.

Hypothesis four has been confirmed. We assume that there is significant relationship between the incidence of hypovitaminosis D and the incidence of endocrine disease. Relationship between vitamin $\mathrm{D}$ and obesity has been confirmed by several studies in which a correlation between higher body fat levels at higher serum vitamin D levels and low dietary calcium intake has been proved. Increased levels of vitamin D can stimulate lipogenesis and suppress lipolysis and consequently increase body fat mass. Recent studies point to an inverse relationship between 
calcium and vitamin D and body fat content. The study confirmed a negative relationship between BMI and vitamin D. Participants with BMI higher than 39.9 were shown to have up to $24 \%$ lower levels of $25(\mathrm{OH}) \mathrm{D}$; up to $18 \%$ lower levels of vitamin D compared to survey participants whose BMI values were up to 25.46. PTH activity can be suppressed by a high supply of calcium and vitamin D; a lower supply of salt and phosphorus. Such suppressed PTH activity may affect insulin sensitivity and the amount of adipose tissue, which affects body weight regulation (8). A study on the effect of vitamin D and calcium on an adipocyte suggests that low calcium intake increases vitamin D level, which subsequently stimulates calcium and is a signal for insulin release. Eventually, fatty acid synthesis is activated and lipolysis is suppressed, leading to weight gain and an increase in adipose tissue. By supplying calcium into the diet, this process is altered in favor of fat mass reduction. Changes in lipid profile occur due to insufficient levels of vitamin D, calcium and vitamin D significantly affect lipid metabolism (2).

Based on the research, it would be appropriate to search for risk groups of patients, especially those with the presence of autoimmune chronic diseases. It would also be appropriate to educate and encourage parents to increase responsibility for the health of their children, informing them about the recommended way of tanning, which is effective for the synthesis of provitamin D and for penetration of UVB rays into the subcutaneous tissue.

\section{Conclusion}

Currently, vitamin D deficiency is a frequently discussed topic worldwide. The extensive effects of vitamin D on the body at its insufficient concentration in the body are associated with the occurrence of diseases. Due to modern times, new lifestyle, diet, and the obesity epidemic, the deficiency of vitamin D has developed into a pandemic of vitamin D deficiency over the past 20 years. Its effects were underestimated only to the level of effects on the musculoskeletal system and locomotor apparatus in the fight against rickets.

At present, there is available surprising new knowledge about the effect of vitamin D on immunity, the incidence of cardiovascular diseases, theeffect on autoimmune diseasesdevelopment, the beneficial effects on weight reduction and many other effects. Due to the prevalence of lifestyle diseases, on which vitamin D has beneficial effects, we can regret that it has been forgotten, and currently we must face its deficiency in both, children and the elderly. Especially the period from birth to infancy and the period of adolescence is at risk for the occurrence of hypovitaminosis D.

To ensure a sustainable optimal concentration of vitamin $\mathrm{D}$, it is most important to bring daylight and physical activity back to our lives. Within prevention we have to search for risk groups of patients and in children cultivate a relationship to regular physical activity from their early age.

\section{References}

1. HOLICK M F, CHEN T C (2008) Vitamin D deficiency: a worldwide problem with health consequences. In The American Journal of Clinical Nutrition [online]. 2008, Vol.87, No. 4, pp. 1080-1086. [cit. 2018-10-10]. Available online: <http://ajcn.nutrition.org/ content/87/4/1080S.long >. ISSN 1938-3207.

2. SASINKA M A, FURKOVA K (2012) The sunshine vitamin: A vitamin D deficiency pandemic. 1. Ed. Bratislava: Herba, 2012. p. 184. ISBN 978-80-89171-90-3.

3. COWAN F J et al. (1997) Inflamatory bowel disease and predisposition to ostopenia. Arch. Dis. Chil. 76, p. 325-329.

4. SEARING D A, LEUNG D Y M (2010) Vitamin D in Atopic Dermatitis, Asthma and Allergic Diseases. In Immunology and Allergy Clinics of North America[online]. 2010, Vol. 30, No. 3, pp. 397-409. [cit. 2018-1013]. Available online: <http://www.ncbi. nlm.nih.gov/pmc/articles/PMC2914320/?tool $=$ pubmed $>$. ISSN 15578607.

5. ANAIZI N (2010) Rediscovering vitamin D. In Libyan Journal of Medicine [online]. 2010, Vol. 5 [cit. 2018-09-25]. Available online: <http://www.ncbi.nlm.nih.gov/pmc/articles/PMC3066777/?tool=pubmed $>$. ISSN 18196357.

6. POPOVICOVA M (2019) The contribution of nurses in breast feeding from the perspective of new mothers, In SUPINOVA, M., FRCOVA, B.: Proceedings of scientific works, 
Multidimensionality in childcare, Collegium Humanum - Warsaw management university 2019, pp. 105 - 121, ISBN 978-83-9529512-6

7. RABATINOVA E (2018) Vitamin D and its deficiency in pediatric patients: diploma thesis. Bratislava: St. Elizabeth University of Health and Social Sciences, Bratislava - Institute of Social Sciences and Health Care of Blessed P. P. Gojdic and V. Hopko, Spisska Nova Ves - Presov. 2018. p. 74.

8. MCCARTY M F \& THOMAS C A (2003) PTH excess may promote weight gain by impeding catecholamine - induced lipolysis implications for the impact of calcium, vitamin D, and alcohol on body weight. Medical hypotheses, vol. 61, no. 5-6, p. 535-542 [44].

9. SOARES M , CHAN SHE PING-DELFOS W, GHANBARI M H (2011) Calcium and vitamin D for obesity: a review of randomized controlled trials. European journal of clinical nutrition, vol. 65, no. 9, pp. 994-1004. 